\title{
An efficient one-pot synthesis and in vitro antimicrobial activity of new pyridine derivatives bearing the tetrazoloquinoline nucleus
}

\author{
Divyesh C. Mungra, Manish P. Patel, and Ranjan G. Patel* \\ Department of Chemistry, S. P. University, Vallabh Vidyanagar-388 120, Gujarat, India \\ E-mail: patelranjanben@yahoo.com
}

\begin{abstract}
A new series of 2-amino-3-cyano-4-tetrazoloquinolinylpyridine derivatives has been synthesized by the one-pot cyclocondensation reaction of a tetrazolo[1,5-a]quinoline-4-carbaldehyde, malononitrile, a heterocyclic/aromatic methyl ketone and ammonium acetate. All the synthesized compounds were subjected to in vitro antimicrobial screening against a panel of pathogenic strains of bacteria and fungi. Some of the compounds were found to be equipotent or more potent than commercial antibiotics as evident from the results.
\end{abstract}

Keywords: 2-Amino-3-cyano-4-tetrazoloquinolinylpyridine, tetrazolo[1,5-a]quinoline-4carbaldehyde, one-pot synthesis, antimicrobial activity, MIC

\section{Introduction}

Many naturally occurring and synthetic compounds bearing pyridine scaffold possess interesting biological properties. ${ }^{1}$ In association with those, 2-amino-3-cyanopyridine derivatives have been identified as IKK- $\beta$ inhibitors ${ }^{2}$ along with its importance and utility as intermediates in preparing variety of heterocyclic compounds. ${ }^{3}$ Consequently, the synthesis of 2-amino-3-cyanopyridine derivatives keeps on attracting much interest in organic chemistry. Various routes for the synthesis of 2-amino-3-cyanopyridine derivatives have been reported using two-component as well as three-component reactions. ${ }^{4-7} \mathrm{Tu}$ and coworkers have reported a facile synthesis of 2amino-3-cyanopyridine derivatives in a one-pot reaction using aromatic aldehyde, methyl ketone, malononitrile and ammonium acetate. ${ }^{4}$ A literature survey shows that a number of pyridine derivatives have been synthesized using various aldehydes but not a single reference have been found where tetrazolo[1,5-a]quinoline-4-carbaldehyde is used. We wish to report herein this heterocyclic aldehyde which is biologically active ${ }^{8-10}$ with a view to obtaining more active heterocyclic system containing two biologically active moieties quinoline ${ }^{11-13}$ and pyridine ${ }^{14,15}$ together. The most suitable protocol for the synthesis of functionalized organic compounds would be a one-pot reaction due to the fact that the synthesis can be performed without the 
isolation of the intermediates, without discharging any functional groups in short reaction time. ${ }^{16}$ Hence, in the present investigation, we report an efficient one-pot multicomponent synthesis of 2-amino-3-cyanopyridine derivatives having tetrazoloquinoline nucleus which have also been recognized as promising new scaffold to endow good biological properties ${ }^{17,18}$ such as antiinflammatory and antimicrobial activity. ${ }^{19-22}$

\section{Results and Discussion}

In the present study, an effort has been made to undertake the synthesis of 2-amino-6-het/aryl-4(7-(un)-substituted-(tetrazolo[1,5-a] quinolin-4-yl))pyridine-3-carbonitriles through a one step process. For this purpose, the required tetrazolo[1,5-a]quinoline derivatives 1a-c were prepared from 2-chloro-3-formylquinoline and sodium azide by our known literature process. ${ }^{23}$ The target compounds 4a-x were prepared in moderate to good yield $(52-77 \%)$ by the reaction of tetrazolo[1,5-a]quinoline-4-carbaldehyde 1a-c, malononitrile 2, (het)aryl methyl ketone 3a-h and ammonium acetate in absolute alcohol (Scheme 1). The formation of compounds $\mathbf{4 a - x}$ may proceed via imine formed from ketone and ammonium acetate, imine reacts with alkylidenemalononitrile formed from Knoevenagel condensation of aldehyde and malononitrile, followed by cycloaddition, isomerization and aromatization to afford the 2-amino-3-cyano-4tetrazoloquinolinylpyridine derivatives 4a-x. The identity of the product was determined by IR, ${ }^{1} \mathrm{H}$ NMR, and ${ }^{13} \mathrm{C}$ NMR spectral studies. The IR spectrum of compound $4 \mathbf{a}$ exhibited absorption at $3410 \mathrm{~cm}^{-1}$ (asymmetric N-H stretching) and $3314 \mathrm{~cm}^{-1}$ (symmetric N-H stretching) for $-\mathrm{NH}_{2}$, $2214 \mathrm{~cm}^{-1}$ for $-\mathrm{CN}, 3015 \mathrm{~cm}^{-1}$ for (aromatic C-H stretching) and 1400 to $1600 \mathrm{~cm}^{-1}$ for $(\mathrm{C}=\mathrm{C}$ aromatic and $\mathrm{C}=\mathrm{N}$ stretching of pyridine). The ${ }^{1} \mathrm{H}$ NMR spectra of compound $4 \mathbf{a}$ showed the absence of the aldehyde proton, moreover singlets at $\delta 7.06 \mathrm{ppm}$ and multiplets at $\delta 7.20-8.71$ ppm appeared for amine and aromatic protons respectively. The ${ }^{13} \mathrm{C}$ NMR spectrum is in good agreement with the structure assigned. All the aromatic carbons of compounds $4 \mathbf{a}$ showed signals around $\delta 115.7-154.3 \mathrm{ppm}$ in the ${ }^{13} \mathrm{C}$ NMR spectra. The signal at $\delta 96.0 \mathrm{ppm}$ is assigned to carbon attached with carbonitrile. Besides, the structure of the compound was well confirmed by its mass spectral studies. Mass spectra of compound $\mathbf{4 i}$ and $\mathbf{4 1}$ gave molecular ion peak at $\mathrm{m} / \mathrm{z} 378$ $(\mathrm{M}+1)$ and $m / z 368(\mathrm{M}+1)$ corresponding to molecular formula $\mathrm{C}_{22} \mathrm{H}_{15} \mathrm{~N}_{7}$ and $\mathrm{C}_{20} \mathrm{H}_{13} \mathrm{~N}_{7} \mathrm{O}$ respectively (Scheme 1). The elemental analysis values are in good agreement with theoretical data. Similarly, all these compounds were characterized on the basis of spectral studies. All the compounds were screened for their antibacterial and antifungal activity. 


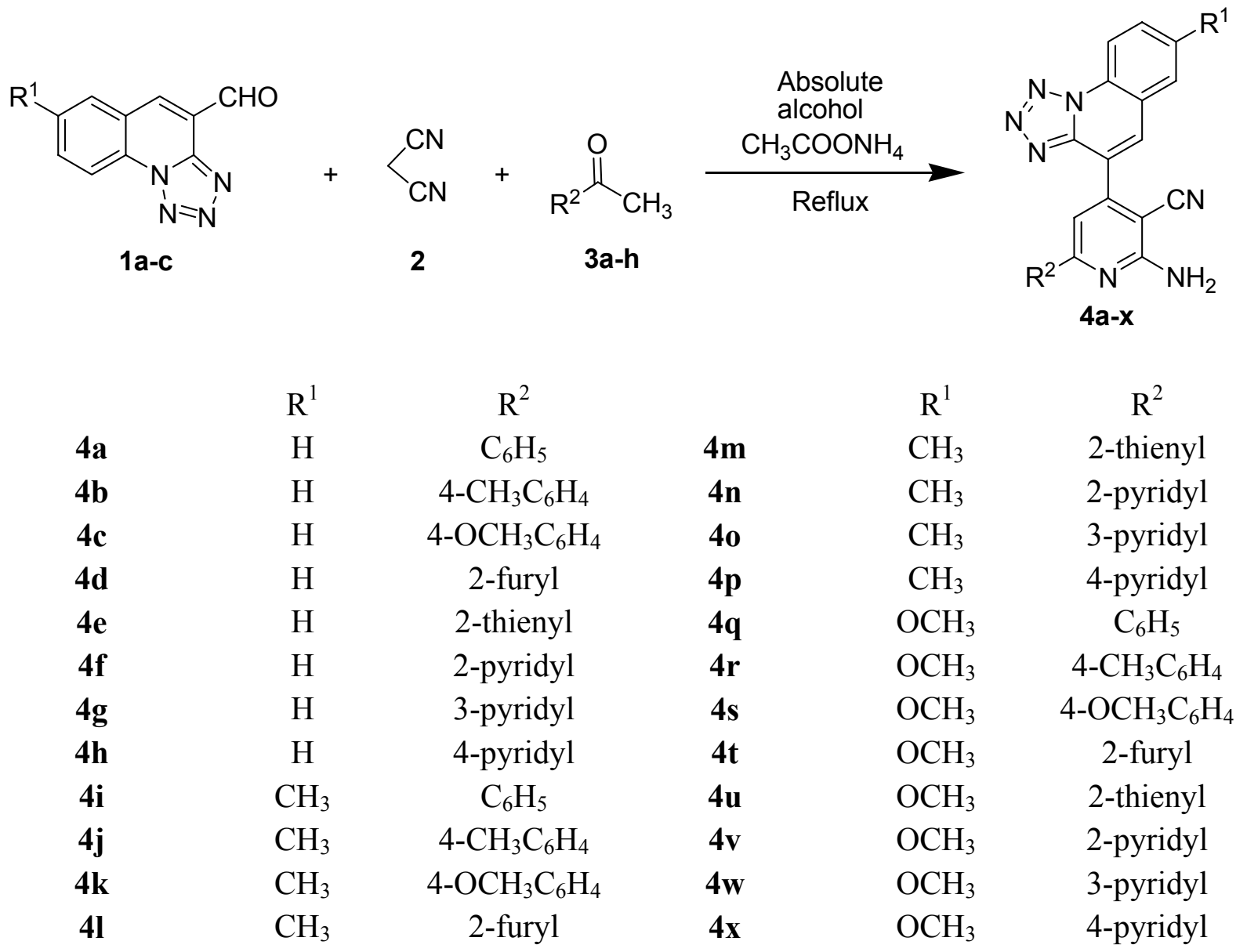

Scheme 1. General synthetic route of 2-amino-3-cyano-4-tetrazoloquinolinylpyridine 4a-x.

\section{Antimicrobial activity}

The in vitro antimicrobial activity of all the synthesized compounds was carried out by broth microdilution method. ${ }^{24}$ Mueller Hinton broth was used as nutrient medium to grow and dilute the compound suspension for the test bacteria and Sabouraud Dextrose broth used for fungal nutrition. Inoculum size for test strain was adjusted to $10^{8} \mathrm{CFU}$ [Colony Forming Unit] per milliliter by comparing the turbidity. The strains employed for the activity were procured from [ MTCC - Micro Type Culture Collection ] Institute of Microbial Technology, Chandigarh.

The compounds 4a-x were screened for their antibacterial activity against Bacillus subtilis (MTCC 441), Clostridium tetani (MTCC 449), Streptococcus pneumoniae (MTCC 1936), Escherichia coli (MTCC 443), Salmonella typhi (MTCC 98), Vibrio cholerae (MTCC 3906) as well as antifungal activity against Aspergillus fumigatus (MTCC 3008) and Candida albicans (MTCC 227). DMSO was used as vehicle to get desired concentration of compounds to test upon microbial strains. The lowest concentration, which showed no visible growth after spot subculture was considered as MIC for each compound. The standard antibiotics used for 
comparison in the present study were ampicillin for evaluating antibacterial activity as well as griseofulvin and nystatin for antifungal activity. The protocols are summarized in (Table 1).

Table 1. Antimicrobial activity of compounds $\mathbf{4 a - x}$

\begin{tabular}{|c|c|c|c|c|c|c|c|c|}
\hline \multicolumn{9}{|c|}{ Minimum inhibitory concentration in $\mu \mathrm{g} / \mathrm{mL}$} \\
\hline & \multicolumn{3}{|c|}{ Gram positive bacteria } & \multicolumn{3}{|c|}{ Gram negative bacteria } & \multicolumn{2}{|c|}{ Fungi } \\
\hline Compd. & $\begin{array}{c}\text { Bacillus } \\
\text { subtilis } \\
\text { MTCC } \\
441\end{array}$ & $\begin{array}{c}\text { Clostridium } \\
\text { tetani } \\
\text { MTCC } \\
449\end{array}$ & $\begin{array}{c}\text { Streptococcus } \\
\text { pneumoniae } \\
\text { MTCC } \\
1936\end{array}$ & $\begin{array}{c}\text { Escherichia } \\
\text { coli } \\
\text { MTCC } \\
443\end{array}$ & $\begin{array}{c}\text { Salmonella } \\
\text { typhi } \\
\text { MTCC } \\
98\end{array}$ & $\begin{array}{c}\text { Vibrio } \\
\text { cholerae } \\
\text { MTCC } \\
3906\end{array}$ & $\begin{array}{c}\text { Aspergillus } \\
\text { fumigatus } \\
\text { MTCC } \\
3008\end{array}$ & $\begin{array}{c}\text { Candida } \\
\text { albicans } \\
\text { MTCC } \\
227\end{array}$ \\
\hline $4 \mathbf{a}$ & 1000 & 250 & 500 & 125 & 125 & 200 & 1000 & 1000 \\
\hline $4 b$ & 500 & 200 & 100 & 500 & 500 & 500 & 1000 & 500 \\
\hline $4 c$ & 1000 & 150 & 100 & 125 & 500 & 250 & 1000 & 500 \\
\hline 4d & 500 & 250 & 500 & 500 & 500 & 250 & 500 & 250 \\
\hline $4 e$ & 500 & 250 & 500 & 100 & 150 & 500 & 1000 & 500 \\
\hline $4 f$ & 500 & 500 & 200 & 500 & 200 & 100 & $>1000$ & $>1000$ \\
\hline $4 g$ & 250 & 200 & 500 & 250 & 500 & 500 & 250 & 250 \\
\hline $4 h$ & 500 & 500 & 500 & 500 & 500 & 250 & 500 & 500 \\
\hline $4 i$ & 250 & 250 & 250 & 100 & 200 & 250 & $>1000$ & $>1000$ \\
\hline $4 j$ & 250 & 250 & 200 & 100 & 200 & 250 & 500 & 250 \\
\hline $4 k$ & 250 & 125 & 100 & 250 & 500 & 500 & 1000 & $>1000$ \\
\hline 41 & 250 & 100 & 100 & 200 & 150 & 500 & 1000 & 500 \\
\hline $4 m$ & 200 & 500 & 200 & $\underline{62.5}$ & 100 & 100 & 1000 & 500 \\
\hline $4 n$ & 150 & 250 & 250 & 125 & 200 & 250 & $>1000$ & 1000 \\
\hline 40 & 500 & 500 & $\underline{62.5}$ & 125 & 125 & $\underline{62.5}$ & $>1000$ & 1000 \\
\hline $4 p$ & 250 & 500 & $\overline{125}$ & 100 & 150 & $\overline{500}$ & 1000 & 500 \\
\hline $4 q$ & 125 & 250 & 250 & 250 & 125 & 100 & 1000 & 500 \\
\hline $4 r$ & 100 & 500 & 500 & 1000 & 1000 & 500 & $>1000$ & 250 \\
\hline $4 s$ & 1000 & 100 & 500 & 1000 & 500 & 500 & 1000 & 500 \\
\hline $4 t$ & 125 & 500 & 250 & 100 & 100 & 500 & 1000 & 200 \\
\hline $4 u$ & 250 & 500 & 100 & 125 & 200 & 500 & 500 & 500 \\
\hline $4 v$ & 250 & 200 & 500 & 125 & 500 & 250 & 500 & 250 \\
\hline $4 w$ & 125 & 250 & 250 & 100 & $\underline{50}$ & 250 & 1000 & 500 \\
\hline $4 x$ & 500 & 500 & 250 & 500 & 125 & 500 & 1000 & 1000 \\
\hline Ampi. & 250 & 250 & 100 & 100 & 100 & 100 & - & - \\
\hline Grise. & - & - & - & - & - & - & 100 & 500 \\
\hline Nyst. & - & - & - & - & - & - & 100 & 100 \\
\hline
\end{tabular}

Ampi.: Ampicillin, Grise.: Griseofulvin, Nyst.: Nystatin

An examination of the data (Table 1) reveals that amongst all the synthesized compounds 4a-x, compound 4o exhibited excellent activity against Gram positive bacteria Streptococcus pneumoniae and Gram negative bacteria Vibrio cholerae while compounds $\mathbf{4 m}$ and $\mathbf{4 w}$ are found to be highly active against Gram negative bacteria Escherichia coli and Salmonella typhi respectively as compared to standard antibiotic ampicillin.

Compounds $\mathbf{4 m}, \mathbf{4 n}, \mathbf{4 q}, \mathbf{4 r}, \mathbf{4 t}$ and $\mathbf{4 w}$ are found to be more potent as compared to standard antibiotic ampicillin against Gram positive bacteria Bacillus subtilis. In case of Gram positive bacteria Clostridium tetani, compounds $4 \mathbf{b}, \mathbf{4 c}, 4 \mathbf{g}, \mathbf{4 k}, \mathbf{4 l}, 4 \mathbf{s}$ and $4 \mathbf{v}$ are found to be more potent than ampicillin. 
Antifungal study revealed that compounds $4 \mathbf{d}, \mathbf{4 g}, \mathbf{4 j}, \mathbf{4 r}, \mathbf{4 t}$ and $4 \mathbf{v}$ are more potent as compared to standard fungicidal griseofulvin against Candida albicans. Most of the compounds were not found sufficiently potent to inhibit Aspergillus fumigatus.

\section{Conclusions}

A series of some new 2-amino-3-cyano-4-tetrazoloquinolinylpyridine derivatives has been synthesized through a facile one-pot multicomponent reaction. This synthetic strategy allows the construction of relatively complicated nitrogen containing heterocyclic system as well as the introduction of various aromatic and heteroaromatic substitutions into 4- and 6- positions of pyridine. It can be concluded from Table 2 that compound 40 having methyl group on tetrazoloquinoline nucleus and 3-pyridyl substitution on pyridine is highly active against Streptococcus pneumoniae as well as Vibrio cholerae. From the activity data, it is worth mentioning that minor change in molecular configuration of these compounds profoundly influences the activity.

\section{Experimental Section}

General. All the reagents were obtained commercially and used with further purification. All melting points were taken in open capillaries and are uncorrected. The monitoring of the progress of all reactions and homogeneity of the synthesized compounds was carried out by TLC. TLC was run using TLC aluminum sheets silica gel $60 \mathrm{~F}_{254}$ (Merck). Elemental analysis (\% C, H, N) was carried out by Perkin Elmer $2400 \mathrm{CHN}$ elemental analyzer at Sophisticated Instrumentation Centre for Applied Research \& Training (SICART), Vallabh Vidyanagar. IR spectra were recorded on a Shimadzu FTIR 8401 spectrophotometer in $\mathrm{KBr} .{ }^{1} \mathrm{H} \mathrm{NMR}$ and ${ }^{13} \mathrm{C}$ NMR spectra were recorded on a Bruker Avance $400 \mathrm{MHz}$ spectrometer using solvent peak as internal standard. Mass spectra were scanned on a Shimadzu LCMS 2010 spectrometer.

\section{General procedure for the synthesis of 2-amino-6-het/aryl-4-(7-(un)-substituted- (tetrazolo[1,5-a]quinolin-4-yl))pyridine-3-carbonitriles 4a-x}

7-(Un)substituted-tetrazolo[1,5- $a$ ]quinoline-4-carbaldehyde 1a-c (5 mmole), malononitrile 2 ( 5 mmole), (het)aryl methyl ketone 3a-h ( 5 mmole), ammonium acetate (40 mmole) and absolute alcohol $(15 \mathrm{ml})$ were charged in a $50 \mathrm{ml}$ round bottom flask. Then, the reaction mixture was refluxed for 2 to $2.5 \mathrm{hr}$. Progress of reaction was monitored by the TLC. After the completion of reaction, the reaction mixture was cooled to room temperature and stirred for $0.5 \mathrm{hr}$. The resulting solid was collected by filtration and washed well with absolute alcohol to obtain the pure solid sample of product $\mathbf{4 a - x}$. 
2-Amino-6-phenyl-4-(tetrazolo[1,5-a]quinolin-4-yl)pyridine-3-carbonitrile 4a. Yield 72\%, m.p. $264{ }^{\circ} \mathrm{C}$, Anal. Calcd. for $\mathrm{C}_{21} \mathrm{H}_{13} \mathrm{~N}_{7}$ : C 69.41, H 3.60, N 26.98\% Found: C 69.32, H 3.56, N 26.72\%. IR (KBr, cm $\left.{ }^{-1}\right): 3410,3314\left(\mathrm{NH}_{2}\right), 2214(\mathrm{CN}), 3015$ (ArC-H). ${ }^{1} \mathrm{H}$ NMR (400 MHz, DMSO- $\left.d_{6}\right): \delta 7.20-8.71(\mathrm{~m}, 11 \mathrm{H}, \mathrm{Ar}-\mathrm{H}), 7.06\left(\mathrm{~s}, 1 \mathrm{H}, \mathrm{NH}_{2}\right) .{ }^{13} \mathrm{C}$ NMR $\left(400 \mathrm{MHz}, \mathrm{DMSO}-d_{6}\right) \delta$ : 96.0 (ㄷ-CN), 115.7, 116.2, 116.7, 120.0, 122.5, 123.9, 128.9, 129.2, 130.1, 130.4, 130.6, 133.0, $134.9,137.7,143.1,146.9,150.4,154.3$ (Ar-C).

2-Amino-6-(4-methylphenyl)-4-(tetrazolo[1,5-a]quinolin-4-yl)pyridine-3-carbonitrile 4b. Yield 65\%, m.p. $276{ }^{\circ} \mathrm{C}$, Anal. Calcd. for $\mathrm{C}_{22} \mathrm{H}_{15} \mathrm{~N}_{7}$ : C 70.01, H 4.00, N 25.97\% Found: C 69.72, H 3.92, N 25.91\%. IR (KBr, cm $\left.{ }^{-1}\right): 3405,3312\left(\mathrm{NH}_{2}\right), 2210(\mathrm{CN}), 3005$ (ArC-H). ${ }^{1} \mathrm{H}$ NMR (400 $\left.\mathrm{MHz}, \mathrm{DMSO}-d_{6}\right): \delta 2.394\left(\mathrm{~s}, 3 \mathrm{H}, \mathrm{CH}_{3}\right), 7.03\left(\mathrm{~s}, 1 \mathrm{H}, \mathrm{NH}_{2}\right), 7.18-8.71(\mathrm{~m}, 10 \mathrm{H}, \mathrm{Ar}-\mathrm{H}) .{ }^{13} \mathrm{C} \mathrm{NMR}$ $\left(400 \mathrm{MHz}, \mathrm{DMSO}-d_{6}\right) \delta: 21.3\left(\mathrm{CH}_{3}\right), 96.0(\underline{\mathrm{C}}-\mathrm{CN}), 115.8,116.8,119.9,122.5,124.0,129.1$, $130.4,133.0,134.8,138.3,140.7,143.0,146.4,146.8,150.5,154.4,160.4,166.9$ (Ar-C).

2-Amino-6-(4-methoxyphenyl)-4-(tetrazolo[1,5-a]quinolin-4-yl)pyridine-3-carbonitrile 4c. Yield 66\%, m.p. 256-259 ${ }^{\circ} \mathrm{C}$, Anal. Calcd. for $\mathrm{C}_{22} \mathrm{H}_{15} \mathrm{~N}_{7} \mathrm{O}$ : C 67.16, H 3.84, N 24.92\% Found: C 67.13, H 3.71, N 25.01\%. IR (KBr, cm $\left.{ }^{-1}\right): 3400,3310\left(\mathrm{NH}_{2}\right), 2180(\mathrm{CN}), 3000(\mathrm{ArC}-\mathrm{H}) .{ }^{1} \mathrm{H}$ NMR (400 MHz, DMSO- $\left.d_{6}\right): \delta 3.97\left(\mathrm{~s}, 3 \mathrm{H}, \mathrm{OCH}_{3}\right), 7.10\left(\mathrm{~s}, 1 \mathrm{H}, \mathrm{NH}_{2}\right), 7.30-8.51(\mathrm{~m}, 10 \mathrm{H}, \mathrm{Ar}-\mathrm{H})$. ${ }^{13} \mathrm{C}$ NMR $\left(400 \mathrm{MHz}, \mathrm{DMSO}-d_{6}\right) \delta: 56.3\left(\mathrm{OCH}_{3}\right), 95.8(\underline{\mathrm{C}}-\mathrm{CN}), 116.8,117.5,119.0,123.8$, $124.9,128.1,131.2,133.0,134.6,138.4,141.7,143.4,146.4,146.2,151.5,154.7,161.4,166.0$ (Ar-C).

2-Amino-6-(2-furyl)-4-(tetrazolo[1,5-a]quinolin-4-yl)pyridine-3-carbonitrile 4d. Yield 76\%, m.p. $273{ }^{\circ} \mathrm{C}$, Anal. Calcd. for $\mathrm{C}_{19} \mathrm{H}_{11} \mathrm{~N}_{7} \mathrm{O}: \mathrm{C}$ 64.56, H 3.13, N 27.74\% Found: C 64.62, H 3.11, N 27.67\%. IR ( $\left.\mathrm{KBr}, \mathrm{cm}^{-1}\right): 3416,3314\left(\mathrm{NH}_{2}\right), 2190(\mathrm{CN}), 3005$ (ArC-H). ${ }^{1} \mathrm{H}$ NMR (400 MHz, DMSO- $\left.d_{6}\right): \delta 7.42\left(\mathrm{~s}, 2 \mathrm{H}, \mathrm{NH}_{2}\right), 7.52-8.61(\mathrm{~m}, 9 \mathrm{H}, \mathrm{Ar}-\mathrm{H}) .{ }^{13} \mathrm{C}$ NMR $\left(400 \mathrm{MHz}, \mathrm{DMSO}-d_{6}\right) \delta$ : 94.7 (ㄷ-CN), 116.4, 119.2, 121.7, 124.4, 128.5, 129.6, 130.1, 134.1, 136.5, 137.7, 138.4, 142.5, $146.4,148.5,148.7,151.4,153.5,154.5$ (Ar-C).

2-Amino-6-(2-thienyl)-4-(tetrazolo[1,5-a]quinolin-4-yl)pyridine-3-carbonitrile 4e. Yield 68\%, m.p. 243-245 ${ }^{\circ} \mathrm{C}$, Anal. Calcd. for $\mathrm{C}_{19} \mathrm{H}_{11} \mathrm{~N}_{7} \mathrm{~S}$ : C 61.77, H 3.00, N 26.54\% Found: C 61.66, H 2.98, N 26.49\%. IR (KBr, cm $\left.{ }^{-1}\right): 3414,3312\left(\mathrm{NH}_{2}\right), 2230(\mathrm{CN}), 3016$ (ArC-H). ${ }^{1} \mathrm{H}$ NMR (400 MHz, DMSO- $\left.d_{6}\right): \delta 7.16\left(\mathrm{~s}, 2 \mathrm{H}, \mathrm{NH}_{2}\right), 7.30-8.54(\mathrm{~m}, 9 \mathrm{H}, \mathrm{Ar}-\mathrm{H}) .{ }^{13} \mathrm{C}$ NMR $(400 \mathrm{MHz}, \mathrm{DMSO}-$ $\left.d_{6}\right) \delta: 93.8(\underline{\mathrm{C}}-\mathrm{CN}), 115.4,117.2,120.7,123.4,126.9,129.0,130.1,133.5,136.5,137.0,137.4$, $141.5,144.8,147.5,148.4,150.4,153.8,155.1$ (Ar-C).

2-Amino-6-(2-pyridyl)-4-(tetrazolo[1,5-a]quinolin-4-yl)pyridine-3-carbonitrile 4f. Yield $77 \%$, m.p. $265{ }^{\circ} \mathrm{C}$, Anal. Calcd. for $\mathrm{C}_{20} \mathrm{H}_{12} \mathrm{~N}_{8}$ : C 65.92, H 3.32, N 30.75\% Found: C 66.08, H 3.24, N 30.61\%. IR (KBr, cm $\left.{ }^{-1}\right): 3416,3330\left(\mathrm{NH}_{2}\right), 2234(\mathrm{CN}), 3028$ (ArC-H). ${ }^{1} \mathrm{H}$ NMR (400 MHz, DMSO- $\left.d_{6}\right): \delta 7.26\left(\mathrm{~s}, 2 \mathrm{H}, \mathrm{NH}_{2}\right), 7.40-9.00(\mathrm{~m}, 10 \mathrm{H}, \mathrm{Ar}-\mathrm{H}) .{ }^{13} \mathrm{C}$ NMR $(400 \mathrm{MHz}, \mathrm{DMSO}-$ $\left.d_{6}\right) \delta: 98.1(\underline{\mathrm{C}}-\mathrm{CN}), 116.7,117.6,122.7,124.4,125.5,128.8,130.9,132.1,135.8,136.6,137.2$, $142.7,146.7,148.2,148.6,150.3,152.2,157.9,158.5$ (Ar-C).

2-Amino-6-(3-pyridyl)-4-(tetrazolo[1,5-a]quinolin-4-yl)pyridine-3-carbonitrile 4g. Yield 69\%, m.p. 270-273 ${ }^{\circ} \mathrm{C}$, Anal. Calcd. for $\mathrm{C}_{20} \mathrm{H}_{12} \mathrm{~N}_{8}$ : C 65.92, H 3.32, N 30.75\% Found: C 66.02, H 3.29, N 30.66\%. IR (KBr, cm $\left.{ }^{-1}\right): 3410,3334\left(\mathrm{NH}_{2}\right), 2216(\mathrm{CN}), 3020(\mathrm{ArC}-\mathrm{H}) .{ }^{1} \mathrm{H}$ NMR (400 
MHz, DMSO- $\left.d_{6}\right): \delta 7.22\left(\mathrm{~s}, 2 \mathrm{H}, \mathrm{NH}_{2}\right), 7.46-9.07(\mathrm{~m}, 10 \mathrm{H}, \mathrm{Ar}-\mathrm{H}) .{ }^{13} \mathrm{C}$ NMR $(400 \mathrm{MHz}, \mathrm{DMSO}-$ $\left.d_{6}\right) \delta: 98.9(\underline{\mathrm{C}}-\mathrm{CN}), 115.4,118.2,120.7,123.4,126.5,128.0,130.0,133.1,135.5,136.3,138.5$, $143.5,147.4,148.5,148.9,151.3,153.2,156.1,158.2$ (Ar-C).

2-Amino-6-(4-pyridyl)-4-(tetrazolo[1,5-a]quinolin-4-yl)pyridine-3-carbonitrile $4 \mathrm{~h}$. Yield $72 \%$, m.p. $258-260{ }^{\circ} \mathrm{C}$, Anal. Calcd. for $\mathrm{C}_{20} \mathrm{H}_{12} \mathrm{~N}_{8}$ : C 65.92, H 3.32, N 30.75\% Found: C 66.04, H 3.35, N 30.67\%. IR (KBr, cm $\left.{ }^{-1}\right): 3400,3336\left(\mathrm{NH}_{2}\right), 2208(\mathrm{CN}), 3005$ (ArC-H). ${ }^{1} \mathrm{H}$ NMR (400 MHz, DMSO- $\left.d_{6}\right): \delta 7.30\left(\mathrm{~s}, 2 \mathrm{H}, \mathrm{NH}_{2}\right), 7.45-8.91(\mathrm{~m}, 10 \mathrm{H}, \mathrm{Ar}-\mathrm{H}) .{ }^{13} \mathrm{C}$ NMR $(400 \mathrm{MHz}, \mathrm{DMSO}-$ $\left.d_{6}\right) \delta: 97.0(\underline{\mathrm{C}}-\mathrm{CN}), 119.5,121.4,123.0,126.7,128.6,129.7,131.4,136.4,138.7,141.0,143.1$, $145.5,146.9,148.4,149.5,151.2,153.1$ (Ar-C).

2-Amino-6-phenyl-4-(7-methyl-(tetrazolo[1,5-a]quinolin-4-yl))pyridine-3-carbonitrile 4i. Yield 71\%, m.p. $274{ }^{\circ} \mathrm{C}$, Anal. Calcd. for $\mathrm{C}_{22} \mathrm{H}_{15} \mathrm{~N}_{7}$ : C 70.01, H 4.00, N 25.97\% Found: C 69.94, H 3.92, N 25.89\%. IR (KBr, cm $\left.{ }^{-1}\right): 3400,3310\left(\mathrm{NH}_{2}\right), 2214(\mathrm{CN}), 3032$ (ArC-H). ${ }^{1} \mathrm{H}$ NMR (400 $\left.\mathrm{MHz}, \mathrm{DMSO}-d_{6}\right): \delta 2.50\left(\mathrm{~s}, 3 \mathrm{H}, \mathrm{CH}_{3}\right), 7.03-8.60(\mathrm{~m}, 10 \mathrm{H}, \mathrm{Ar}-\mathrm{H}) .{ }^{13} \mathrm{C}$ NMR $(400 \mathrm{MHz}, \mathrm{DMSO}-$ $\left.d_{6}\right) \delta: 21.3\left(\mathrm{CH}_{3}\right), 96.0(\underline{\mathrm{C}}-\mathrm{CN}), 115.8,119.4,121.5,126.4,126.9,128.2,130.9,132.1,134.4$, 136.5, 138.8, 140.5, 144.9, 147.8, 149.3, 150.5, 153.4, 154.4 (Ar-C), MS: (M+1) 378.

2-Amino-6-(4-methylphenyl)-4-(7-methyl-(tetrazolo[1,5-a]quinolin-4-yl))pyridine-3carbonitrile 4j. Yield 72\%, m.p. $264{ }^{\circ} \mathrm{C}$, Anal. Calcd. for $\mathrm{C}_{23} \mathrm{H}_{17} \mathrm{~N}_{7}$ : C 70.57, H 4.37, N 25.04\% Found: C 70.42, H 4.25, N 25.01\%. IR $\left(\mathrm{KBr}, \mathrm{cm}^{-1}\right)$ : 3405, $3300\left(\mathrm{NH}_{2}\right), 2240(\mathrm{CN}), 3026(\mathrm{ArC}--$ H). ${ }^{1} \mathrm{H}$ NMR (400 MHz, DMSO- $\left.d_{6}\right): \delta 2.39\left(\mathrm{~s}, 3 \mathrm{H}, \mathrm{CH}_{3}\right), 2.45\left(\mathrm{~s}, 3 \mathrm{H}, \mathrm{CH}_{3}\right), 7.03\left(\mathrm{~s}, 2 \mathrm{H}, \mathrm{NH}_{2}\right)$, 7.18-8.71 (m, 9H, Ar-H). ${ }^{13} \mathrm{C}$ NMR (400 MHz, DMSO- $\left.d_{6}\right) \delta: 21.3\left(\mathrm{CH}_{3}\right), 23.4\left(\mathrm{CH}_{3}\right), 96.0(\underline{\mathrm{C}}-$ $\mathrm{CN}), 115.8,119.9,122.5,124.0,128.9,130.4,130.6,133.0,133.3,138.8,139.9,140.7,145.0$, $146.4,146.8,150.5,154.4,160.4$ (Ar-C).

2-Amino-6-(4-methoxyphenyl)-4-(7-methyl-(tetrazolo[1,5-a]quinolin-4-yl))pyridine-3carbonitrile 4k. Yield 70\%, m.p. $243{ }^{\circ} \mathrm{C}$, Anal. Calcd. for $\mathrm{C}_{23} \mathrm{H}_{17} \mathrm{~N}_{7} \mathrm{O}: \mathrm{C}$ 67.80, H 4.20, N 24.06\% Found: C 67.77, H 4.11, N 24.00\%. IR (KBr, cm $\left.{ }^{-1}\right): 3436,3310\left(\mathrm{NH}_{2}\right), 2210(\mathrm{CN}), 3005$ (ArC-H). ${ }^{1} \mathrm{H}$ NMR (400 MHz, DMSO- $\left.d_{6}\right): \delta 2.50$ (s, 3H, CH $), 3.97$ (s, 3H, $\left.\mathrm{OCH}_{3}\right), 6.87$ (s, 2H, $\left.\mathrm{NH}_{2}\right), 7.03-8.62(\mathrm{~m}, 9 \mathrm{H}, \mathrm{Ar}-\mathrm{H}) .{ }^{13} \mathrm{C} \mathrm{NMR}\left(400 \mathrm{MHz}, \mathrm{DMSO}-d_{6}\right) \delta: 21.3\left(\mathrm{CH}_{3}\right), 56.3\left(\mathrm{OCH}_{3}\right)$, 96.4 (ㄷ-CN), 117.1, 121.9, 124.4, 126.7, 128.3, 128.9, 129.1, 132.7, 133.3, 136.0, 137.4, 138.6, $141.5,143.0,144.2,145.8,148.0,150.4$ (Ar-C).

2-Amino-6-(2-furyl)-4-(7-methyl-(tetrazolo[1,5-a]quinolin-4-yl))pyridine-3-carbonitrile 41. Yield 73\%, m.p. 278-280 ${ }^{\circ} \mathrm{C}$, Anal. Calcd. for $\mathrm{C}_{20} \mathrm{H}_{13} \mathrm{~N}_{7} \mathrm{O}: \mathrm{C} 65.38, \mathrm{H} 3.56, \mathrm{~N} 26.68 \%$ Found: C 65.25, H 3.44, N 26.65\%. IR (KBr, cm $\left.{ }^{-1}\right): 3410,3336\left(\mathrm{NH}_{2}\right), 2214(\mathrm{CN}), 3030$ (ArC-H). ${ }^{1} \mathrm{H}$ NMR (400 MHz, DMSO- $\left.d_{6}\right): \delta 2.50\left(\mathrm{~s}, 3 \mathrm{H}, \mathrm{CH}_{3}\right), 6.79\left(\mathrm{~s}, 2 \mathrm{H}, \mathrm{NH}_{2}\right), 7.03-8.63(\mathrm{~m}, 8 \mathrm{H}, \mathrm{Ar}-\mathrm{H})$. ${ }^{13} \mathrm{C}$ NMR (400 MHz, DMSO- $\left.d_{6}\right) \delta: 20.8\left(\mathrm{CH}_{3}\right), 96.7(\underline{\mathrm{C}}-\mathrm{CN}), 119.1,120.4,122.9,124.0,126.8$, $126.9,128.7,130.0,131.7,133.2,135.1,136.3,138.5,139.9,140.5,141.7,142.9,146.1$ (Ar-C), MS: $(\mathrm{M}+1) 368$.

2-Amino-6-(2-thienyl)-4-(7-methyl-(tetrazolo[1,5-a]quinolin-4-yl))pyridine-3-carbonitrile 4m. Yield 66\%, m.p. 264-267 ${ }^{\circ} \mathrm{C}$, Anal. Calcd. for $\mathrm{C}_{20} \mathrm{H}_{13} \mathrm{~N}_{7} \mathrm{~S}: \mathrm{C} 62.64, \mathrm{H} 3.41, \mathrm{~N} 25.57 \%$ Found: C 62.60, H 3.39, N 25.42\%. IR (KBr, cm $\left.{ }^{-1}\right)$ : 3400, $3316\left(\mathrm{NH}_{2}\right), 2230(\mathrm{CN}), 3005$ (ArC-H). ${ }^{1} \mathrm{H}$ NMR (400 MHz, DMSO- $\left.d_{6}\right): \delta 2.47\left(\mathrm{~s}, 3 \mathrm{H}, \mathrm{CH}_{3}\right), 6.84\left(\mathrm{~s}, 2 \mathrm{H}, \mathrm{NH}_{2}\right), 6.96-8.54(\mathrm{~m}, 8 \mathrm{H}$, 
Ar-H). ${ }^{13} \mathrm{C}$ NMR (400 MHz, DMSO- $\left.d_{6}\right) \delta: 21.6\left(\mathrm{CH}_{3}\right), 94.1$ ( $\left.\underline{\mathrm{C}}-\mathrm{CN}\right), 114.1,118.4,122.6,124.0$, $126.0,127.9,128.7,130.4,131.1,134.2,135.1,136.0,137.5,138.9,141.0,141.7,142.4,143.8$ (Ar-C).

2-Amino-6-(2-pyridyl)-4-(7-methyl-(tetrazolo[1,5-a]quinolin-4-yl))pyridine-3-carbonitrile 4n. Yield 70\%, m.p. $252{ }^{\circ} \mathrm{C}$, Anal. Calcd. for $\mathrm{C}_{21} \mathrm{H}_{14} \mathrm{~N}_{8}$ : C 66.65, H 3.72, N 29.61\% Found: C 65.70, H 3.64, N 29.58\%. IR (KBr, cm $\left.{ }^{-1}\right): 3430,3326\left(\mathrm{NH}_{2}\right), 2230(\mathrm{CN}), 3016(\mathrm{ArC}-\mathrm{H}) .{ }^{1} \mathrm{H}$ NMR (400 MHz, DMSO- $\left.d_{6}\right): \delta 2.21\left(\mathrm{~s}, 3 \mathrm{H}, \mathrm{CH}_{3}\right), 6.94\left(\mathrm{~s}, 2 \mathrm{H}, \mathrm{NH}_{2}\right), 7.23-8.78(\mathrm{~m}, 9 \mathrm{H}, \mathrm{Ar}-\mathrm{H})$. ${ }^{13} \mathrm{C}$ NMR (400 MHz, DMSO- $\left.d_{6}\right) \delta: 21.9\left(\mathrm{CH}_{3}\right), 94.8(\underline{\mathrm{C}}-\mathrm{CN}), 115.6,118.3,121.4,123.7,125.5$, $127.4,130.0,133.4,133.9,134.7,135.6,137.2,139.0,140.4,144.6,148.3,152.0,153.4,154.9$ (Ar-C).

2-Amino-6-(3-pyridyl)-4-(7-methyl-(tetrazolo[1,5-a]quinolin-4-yl))pyridine-3-carbonitrile 4o. Yield 54\%, m.p. 270-273 ${ }^{\circ} \mathrm{C}$, Anal. Calcd. for $\mathrm{C}_{21} \mathrm{H}_{14} \mathrm{~N}_{8}$ : C 66.65, H 3.72, N 29.61\% Found: C 65.68, H 3.69, N 29.48\%. IR (KBr, cm $\left.{ }^{-1}\right): 3400,3330\left(\mathrm{NH}_{2}\right), 2216(\mathrm{CN}), 3000(\mathrm{ArC}-\mathrm{H}) .{ }^{1} \mathrm{H}$ NMR (400 MHz, DMSO-d $): \delta 2.25\left(\mathrm{~s}, 3 \mathrm{H}, \mathrm{CH}_{3}\right), 7.04$ (s, 2H, NH ), 7.20-8.70 (m, 9H, Ar-H). ${ }^{13} \mathrm{C}$ NMR (400 MHz, DMSO- $\left.d_{6}\right) \delta: 22.1\left(\mathrm{CH}_{3}\right), 95.6(\underline{\mathrm{C}}-\mathrm{CN}), 117.1,119.3,122.9,123.0,126.5$, $128.4,130.5,133.0,133.9,134.5,136.7,137.0,139.3,141.5,144.0,148.1,152.3,154.2,155.0$ (Ar-C).

\section{2-Amino-6-(4-pyridyl)-4-(7-methyl-(tetrazolo[1,5-a]quinolin-4-yl))pyridine-3-carbonitrile}

4p. Yield 68\%, m.p. $267{ }^{\circ} \mathrm{C}$, Anal. Calcd. for $\mathrm{C}_{21} \mathrm{H}_{14} \mathrm{~N}_{8}$ : C 66.65, H 3.72, N 29.61\% Found: C 65.61, H 3.80, N 29.55\%. IR (KBr, cm $\left.{ }^{-1}\right): 3408,3338\left(\mathrm{NH}_{2}\right), 2236(\mathrm{CN}), 3018(\mathrm{ArC}-\mathrm{H}) .{ }^{1} \mathrm{H}$ NMR (400 MHz, DMSO-d $): \delta 2.20\left(\mathrm{~s}, 3 \mathrm{H}, \mathrm{CH}_{3}\right), 7.12\left(\mathrm{~s}, 2 \mathrm{H}, \mathrm{NH}_{2}\right), 7.26-8.77(\mathrm{~m}, 9 \mathrm{H}, \mathrm{Ar}-\mathrm{H})$. ${ }^{13} \mathrm{C}$ NMR (400 MHz, DMSO- $\left.d_{6}\right) \delta: 21.8\left(\mathrm{CH}_{3}\right), 94.2(\underline{\mathrm{C}}-\mathrm{CN}), 117.1,119.3,122.9,123.0,126.5$, $128.4,130.5,133.0,133.9,134.5,136.7,137.0,139.3,141.5,144.0,148.1,152.3$ (Ar-C).

2-Amino-6-phenyl-4-(7-methoxy-(tetrazolo[1,5-a]quinolin-4-yl))pyridine-3-carbonitrile 4q. Yield 62\%, m.p. 243-245 ${ }^{\circ} \mathrm{C}$, Anal. Calcd. for $\mathrm{C}_{22} \mathrm{H}_{15} \mathrm{~N}_{7} \mathrm{O}$ : C 67.16, H 3.84, N 24.92\% Found: C 67.10, H 3.74, N 24.89\%. IR (KBr, cm $\left.{ }^{-1}\right): 3440,3322\left(\mathrm{NH}_{2}\right), 2232(\mathrm{CN}), 3028(\mathrm{ArC}-\mathrm{H}) .{ }^{1} \mathrm{H}$ NMR (400 MHz, DMSO- $\left.d_{6}\right): \delta 3.84\left(\mathrm{~s}, 3 \mathrm{H}, \mathrm{OCH}_{3}\right), 7.01\left(\mathrm{~s}, 1 \mathrm{H}, \mathrm{NH}_{2}\right), 7.16-8.31(\mathrm{~m}, 10 \mathrm{H}, \mathrm{Ar}-\mathrm{H})$. ${ }^{13} \mathrm{C}$ NMR (400 MHz, DMSO- $\left.d_{6}\right) \delta$ : $56.8\left(\mathrm{OCH}_{3}\right), 96.0(\underline{\mathrm{C}}-\mathrm{CN}), 115.7,116.2,116.7,120.0$, $122.5,123.9,128.9,129.2,130.1,130.4,130.6,133.0,134.9,137.7,143.1,146.9,150.4,154.3$ (Ar-C).

2-Amino-6-(4-methylphenyl)-4-(7-methoxy-(tetrazolo[1,5-a]quinolin-4-yl))pyridine-3-

carbonitrile 4r. Yield 70\%, m.p. 274-276 ${ }^{\circ} \mathrm{C}$, Anal. Calcd. for $\mathrm{C}_{23} \mathrm{H}_{17} \mathrm{~N}_{7} \mathrm{O}: \mathrm{C}$ 67.80, H 4.20, N 24.06\% Found: C 67.71, H 4.13, N 23.00\%. IR (KBr, cm $\left.{ }^{-1}\right): 3400,3310\left(\mathrm{NH}_{2}\right), 2210(\mathrm{CN}), 2990$ $(\mathrm{ArC}-\mathrm{H}) .{ }^{1} \mathrm{H}$ NMR $\left(400 \mathrm{MHz}, \mathrm{DMSO}-d_{6}\right): \delta 2.50\left(\mathrm{~s}, 3 \mathrm{H}, \mathrm{CH}_{3}\right), 3.86\left(\mathrm{~s}, 3 \mathrm{H}, \mathrm{OCH}_{3}\right), 6.91(\mathrm{~s}, 2 \mathrm{H}$, $\left.\mathrm{NH}_{2}\right), 7.08-8.68(\mathrm{~m}, 9 \mathrm{H}, \mathrm{Ar}-\mathrm{H}) .{ }^{13} \mathrm{C} \mathrm{NMR}\left(400 \mathrm{MHz}, \mathrm{DMSO}-d_{6}\right) \delta: 21.4\left(\mathrm{CH}_{3}\right), 55.9\left(\mathrm{OCH}_{3}\right)$, 97.2 (ㄷ-CN), 118.1, 120.9, 123.1, 126.7, 128.0, 128.9, 129.6, 133.1, 133.3, 136.4, 138.4, 138.6, $141.9,143.0,144.1,145.3,149.0,152.4$ (Ar-C).

2-Amino-6-(4-methoxyphenyl)-4-(7-methoxy-(tetrazolo[1,5-a]quinolin-4-yl))pyridine-3carbonitrile 4s. Yield 52\%, m.p. $292{ }^{\circ} \mathrm{C}$, Anal. Calcd. for $\mathrm{C}_{23} \mathrm{H}_{17} \mathrm{~N}_{7} \mathrm{O}_{2}: \mathrm{C}$ 65.24, H 4.04, N 23.15\% Found: C 65.30, H 4.00, N 23.11\%. IR (KBr, cm $\left.{ }^{-1}\right): 3420,3312\left(\mathrm{NH}_{2}\right), 2230(\mathrm{CN}), 3020$ 
(ArC-H). ${ }^{1} \mathrm{H}$ NMR (400 MHz, DMSO-d $): \delta 3.90\left(\mathrm{~s}, 3 \mathrm{H}, \mathrm{OCH}_{3}\right), 3.97\left(\mathrm{~s}, 3 \mathrm{H}, \mathrm{OCH}_{3}\right), 6.98(\mathrm{~s}$, $\left.1 \mathrm{H}, \mathrm{NH}_{2}\right), 7.12-8.70(9 \mathrm{H}, \mathrm{m}, \mathrm{Ar}-\mathrm{H}) .{ }^{13} \mathrm{C} \mathrm{NMR}\left(400 \mathrm{MHz}, \mathrm{DMSO}-d_{6}\right) \delta: 54.9\left(\mathrm{OCH}_{3}\right), 56.3$ $\left(\mathrm{OCH}_{3}\right), 95.8(\underline{\mathrm{C}}-\mathrm{CN}), 117.4,121.9,123.3,123.6,126.7,128.9,129.9,129.6,130.3,133.4$, $133.6,136.9,138.4,139.6,141.0,143.5,144.6,145.3$ (Ar-C).

2-Amino-6-(2-furyl)-4-(7-methoxy-(tetrazolo[1,5-a]quinolin-4-yl))pyridine-3-carbonitrile 4t. Yield 70\%, m.p. 266-268 ${ }^{\circ} \mathrm{C}$, Anal. Calcd. for $\mathrm{C}_{20} \mathrm{H}_{13} \mathrm{~N}_{7} \mathrm{O}_{2}$ : C 62.66, H 3.41, N 25.57\% Found: C 62.60, H 3.39, N 25.52\%. IR (KBr, cm $\left.{ }^{-1}\right)$ : 3440, $3326\left(\mathrm{NH}_{2}\right), 2200(\mathrm{CN}), 3035(\mathrm{ArC}--$ H). ${ }^{1} \mathrm{H}$ NMR (400 MHz, DMSO- $\left.d_{6}\right): \delta 3.76\left(\mathrm{~s}, 3 \mathrm{H}, \mathrm{OCH}_{3}\right), 6.98\left(\mathrm{~s}, 2 \mathrm{H}, \mathrm{NH}_{2}\right), 7.41-8.94(\mathrm{~m}, 8 \mathrm{H}$, $\mathrm{Ar}-\mathrm{H}) .{ }^{13} \mathrm{C}$ NMR (400 MHz, DMSO- $\left.d_{6}\right) \delta: 56.1\left(\mathrm{OCH}_{3}\right), 96.3(\underline{\mathrm{C}}-\mathrm{CN}), 116.0,117.8,119.2$, $120.2,122.2$, 124.4, 126.9, 129.3, 131.3, 134.1, 136.0, 138.1, 140.4, 143.8, 144.2, 146.9, 148.9, 152.1 (Ar-C).

2-Amino-6-(2-thienyl)-4-(7-methoxy-(tetrazolo[1,5-a]quinolin-4-yl))pyridine-3-carbonitrile 4u. Yield 71\%, m.p. 271-273 ${ }^{\circ} \mathrm{C}$, Anal. Calcd. for $\mathrm{C}_{20} \mathrm{H}_{13} \mathrm{~N}_{7} \mathrm{OS}$ : C 60.14, H 3.28, N 24.54\% Found: C 60.10, H 3.30, N 25.61\%. IR (KBr, cm $\left.{ }^{-1}\right): 3430,3320\left(\mathrm{NH}_{2}\right), 2220(\mathrm{CN}), 3040(\mathrm{ArC}--$ H). ${ }^{1} \mathrm{H}$ NMR (400 MHz, DMSO- $\left.d_{6}\right): \delta 3.45\left(\mathrm{~s}, 3 \mathrm{H}, \mathrm{OCH}_{3}\right), 7.21\left(\mathrm{~s}, 2 \mathrm{H}, \mathrm{NH}_{2}\right), 7.35-8.83(\mathrm{~m}, 8 \mathrm{H}$, Ar-H). ${ }^{13} \mathrm{C}$ NMR (400 MHz, DMSO- $\left.d_{6}\right) \delta: 55.3\left(\mathrm{OCH}_{3}\right), 96.8(\underline{\mathrm{C}}-\mathrm{CN}), 117.8,118.8,119.2$, $120.7,123.2$, 124.9, 126.5, 129.0, 133.3, 134.5, 136.6, 138.4, 141.4, 143.5, 144.2, 146.0, 148.2, 151.3 (Ar-C).

2-Amino-6-(2-pyridyl)-4-(7-methoxy-(tetrazolo[1,5-a]quinolin-4-yl))pyridine-3-carbonitrile 4v. Yield $60 \%$, m.p. $276{ }^{\circ} \mathrm{C}$, Anal. Calcd. for $\mathrm{C}_{21} \mathrm{H}_{14} \mathrm{~N}_{8} \mathrm{O}: \mathrm{C} 63.95, \mathrm{H} 3.57$, N 28.41\% Found: C 64.02, H 3.60, N 28.38\%. IR (KBr, cm $\left.{ }^{-1}\right): 3410,3318\left(\mathrm{NH}_{2}\right), 2214(\mathrm{CN}), 3000(\mathrm{ArC}-\mathrm{H}) .{ }^{1} \mathrm{H}$ NMR (400 MHz, DMSO-d $\left.d_{6}\right): \delta 3.64\left(\mathrm{~s}, 3 \mathrm{H}, \mathrm{OCH}_{3}\right), 7.21\left(\mathrm{~s}, 2 \mathrm{H}, \mathrm{NH}_{2}\right), 7.33-8.71(\mathrm{~m}, 9 \mathrm{H}, \mathrm{Ar}-\mathrm{H})$. ${ }^{13} \mathrm{C}$ NMR (400 MHz, DMSO- $\left.d_{6}\right) \delta: 55.4\left(\mathrm{OCH}_{3}\right), 96.7(\underline{\mathrm{C}}-\mathrm{CN}), 117.7,118.0,119.4,120.8$, $123.1,125.9,127.8,129.0,131.5,135.4,138.7,140.1,142.7,145.6,146.8,148.1,149.0,150.4$, $153.2($ Ar-C).

2-Amino-6-(3-pyridyl)-4-(7-methoxy-(tetrazolo[1,5-a]quinolin-4-yl))pyridine-3-carbonitrile 4w. Yield 58\%, m.p. 244-247 ${ }^{\circ} \mathrm{C}$, Anal. Calcd. for $\mathrm{C}_{21} \mathrm{H}_{14} \mathrm{~N}_{8} \mathrm{O}: \mathrm{C} 63.95, \mathrm{H} \mathrm{3.57,} \mathrm{N} \mathrm{28.41 \%}$ Found: C 63.88, H 3.57, N 28.41\%. IR (KBr, cm $\left.{ }^{-1}\right)$ : 3416, $3310\left(\mathrm{NH}_{2}\right), 2228(\mathrm{CN}), 3020(\mathrm{ArC}--$ H). ${ }^{1} \mathrm{H}$ NMR (400 MHz, DMSO- $\left.d_{6}\right): \delta 3.72\left(\mathrm{~s}, 3 \mathrm{H}, \mathrm{OCH}_{3}\right), 7.27\left(\mathrm{~s}, 2 \mathrm{H}, \mathrm{NH}_{2}\right), 7.30-8.74(\mathrm{~m}, 9 \mathrm{H}$, Ar-H). ${ }^{13} \mathrm{C}$ NMR (400 MHz, DMSO- $\left.d_{6}\right) \delta: 56.9\left(\mathrm{OCH}_{3}\right), 97.2(\underline{\mathrm{C}}-\mathrm{CN}), 115.7,117.0,118.6$, 120.2 , 124.4, 126.0, 128.8, 129.7, 131.8, 136.4, 138.0, 140.4, 142.5, 143.0, 146.5, 148.8, 149.4, 151.0, 152.8 (Ar-C).

2-Amino-6-(4-pyridyl)-4-(7-methoxy-(tetrazolo[1,5-a]quinolin-4-yl))pyridine-3-carbonitrile 4x. Yield 70\%, m.p. 258-260 ${ }^{\circ} \mathrm{C}$, Anal. Calcd. for $\mathrm{C}_{21} \mathrm{H}_{14} \mathrm{~N}_{8} \mathrm{O}: \mathrm{C}$ 63.95, H 3.57, N 28.41\% Found: C 63.91, H 3.44, N 28.39\%. IR (KBr, cm $\left.{ }^{-1}\right)$ : 3446, $3318\left(\mathrm{NH}_{2}\right), 2220(\mathrm{CN}), 3024(\mathrm{ArC}--$ H). ${ }^{1} \mathrm{H}$ NMR (400 MHz, DMSO- $\left.d_{6}\right): \delta 3.85\left(\mathrm{~s}, 3 \mathrm{H}, \mathrm{OCH}_{3}\right), 7.04\left(\mathrm{~s}, 2 \mathrm{H}, \mathrm{NH}_{2}\right), 7.14-8.81(\mathrm{~m}, 9 \mathrm{H}$, $\mathrm{Ar}-\mathrm{H}) .{ }^{13} \mathrm{C}$ NMR (400 MHz, DMSO- $\left.d_{6}\right) \delta: 56.1\left(\mathrm{OCH}_{3}\right), 97.4(\underline{\mathrm{C}}-\mathrm{CN}), 120.4,122.4,125.3$, $126.9,128.3,130.2,131.0,132.2,133.3,135.9,137.4,138.6,141.6,142.3,144.8,145.5,147.5$ (Ar-C). 


\section{Acknowledgements}

The authors express their sincere thanks to the Department of Chemistry, S P University for providing research facilities, Vaibhav Analytical Laboratory, Ahmedabad for IR spectral analysis as well as Oxygen Healthcare Research Pvt. Ltd., Ahmedabad for providing mass spectrometry facilities. We are also thankful to Microcare Laboratory, Surat for antimicrobial activity.

\section{References}

1. Temple, C.; Rener, Jr. G. A.; Raud, W. R.; Noker, P. E. J. Med. Chem. 1992, 35, 3686.

2. Murata, T.; Shimada, M.; Sakakibara, S.; Yoshino, T.; Kadono, H.; Masuda, T.; Shimazaki, M.; Shintani, T.; Fuchikami, K.; Sakai, K.; Inbe, H.; Takeshita, K.; Niki, T.; Umeda, M.; Bacon, K. B.; Ziegelbauer, K. B.; Lowinger, T. B. Bioorg. Med. Chem. Lett. 2003, 13, 913.

3. (a) Shishoo, C. J.; Devani, M. B.; Bhadti, V. S.; Ananthan, S.; Ullas, G. V. Tetrahedron Lett. 1983, 24, 4611. (b) Doe, K.; Avasthi, K.; Pratap, R.; Bakuni, D. S.; Joshi, M. N. Indian J. Chem. 1990, 29B, 459.

4. Feng, S.; Shujiang, T.; Fang, F.; Tuanjie, L. Arkivoc 2005, (i), 137.

5. Rafat, M. S.; Fathy, F. A. J. Chem. Re. (S). 1997, 294.

6. Vyas, D. H.; Tala, S. D.; Akbari, J. D.; Dhaduk, M. F.; Joshi, K. A. Indian J. Chem. 2009, $48 B, 833$.

7. Zhou, J. F.; Gong, G. X.; Zhu, F. X.; Zhi, S. J. Chin. Chem. Lett. 2009, 20, 37.

8. Dreikorn, B. A. U. S. Patent 3764 681, 1973.

9. Wright, T. L. U. S. Patent 4496 569, 1985.

10. Bekhit, A. A.; El-Sayed, O. A.; Al-Allaf, T. A. K.; Aboul-Enein, H. Y.; Kunhi, M.; Pulicat, S. M.; Al-Hussain, K.; Al-Khodairy, F.; Arif, J. Eur. J. Med. Chem. 2004, 39, 499.

11. Kumar, R. N.; Suresh, T.; Mohan, P. S. Indian J. Chem. 2003, 42B, 688.

12. Moiseev, I. K,; Zemtsova, M. N.; Trakhtenberg, P. L.; Kulikowa, D. A.; Pskobkina, I.; Neschadim, G. N.; Ostapchuk, N. V. Khim.-Farm Zh. 1998, 22, 1448.

13. Strong; Lee, H. Chem. Abstr. 1997, 126, 17876 b.

14. Brahmbhatt, D. I.; Pandya, V. P.; Patel, C. N.; Patel, M. A. Indian J. Chem. 2005, 44B, 1863.

15. Cai, X. M.; Wang, Q. F.; Yan, C. G. Synth. Commun. 2007, 37, 223.

16. Boulard, L.; BouzBouz, S.; Cossy, J.; Franck, X.; Figadere, B. Tetrahedron Lett. 2004, 45, 6603.

17. Mukherjee, A.; Akhtar, M. S.; Sharma, V. L.; Seth, M.; Bhaduri, A. P.; Agnihotri, A.; Mehrotra, P. K.; Kamboj, V. P. J. Med. Chem. 1989, 32, 2297.

18. Gupta, R.; Gupta, A. K.; Paul, S. Indian J. Chem. 2000, 39B, 847. 
19. Bekhit, A. A.; El-Sayed, O. A.; Aboulmagd, E.; Park, J. Y. Eur. J. Med. Chem. 2004, 39, 249.

20. Angibaud, P.; Bourdrez, X.; End, D. W. Bio. \& Med. Chem. 2003, 13, 4365.

21. Bawa, S.; Kumar, S. Indian J. Chem. 2009, 48B, 142.

22. Thota, S.; Argade, A.; Singh, R.; Lu, H. H.; Huang, P., U. S. Patent 7358259B2, 2008.

23. Ladani, N. K.; Patel, M. P.; Patel, R. G. Arkivoc 2009, (vii), 292.

24. National Committee for Clinical Laboratory Standards (NCCLS), 940, West Valley Road, Suite 1400, Wayne, Pennsylvania 19087-1898, USA. Performance Standards for Antimicrobial Susceptibility Testing; Twelfth Informational Supplement (ISBN 1-56238454 -6), 2002, M100-S12 (M7). 